\title{
THE CENTRAL GAS CONSUMPTION TIMESCALE IN SPIRALS
}

\author{
S. JOGEE AND J. D. P. KENNEY
}

Yale University Astronomy Department, New Haven, CT 065208101, U.S.A.

It is now widely accepted that a combination of torques and dissipation can drive molecular gas into the circumnuclear (inner $\mathrm{kpc}$ ) region of spirals. However, the fate of the circumnuclear gas is poorly understood and depends on inflow mechanisms, outflow processes, and on star formation. It is unclear why spiral galaxies convert comparable amount of circumnuclear $\mathrm{H}_{2}$ into stars over a timescale $\left(t_{\mathrm{sf}}\right)$ that varies by nearly two orders of magnitude between galaxies. The ratio $\mathrm{M}\left(\mathrm{H}_{2}\right) / \mathrm{L}(\mathrm{RC})$ can be used to show the range in this timescale, where $\mathrm{L}(\mathrm{RC})$ is the $1.49 \mathrm{GHz}$ radio continuum ( $\mathrm{RC}$ ) luminosity (Condon et al. 1990), and $\mathrm{M}\left(\mathrm{H}_{2}\right)$ the hydrogen mass traced by CO observations in the central $45^{\prime \prime}(4.5 \mathrm{kpc}$ for $\mathrm{D}=20 \mathrm{Mpc})$. A similar range is seen if the central 10 micron luminosity is used. Preliminary results from Jogee's thesis suggest that the circumnuclear gas consumption timescale by star formation $\left(\mathrm{t}_{\mathrm{sf}}\right)$ is correlated with the gas morphology (see Fig. 1) and kinematics. In particular, the value of $\mathrm{t}_{\mathrm{sf}}$ spans a large range in different kinematic regimes within a given galaxy, and together with inflow, this can lead some galaxies to follow evolutionary paths sketched in Fig. 1.

The timescale $t_{s f}$ is short ( $\leq 1 \mathrm{Gyr}$ ) in NGC 3504 (Kenney et al. 1993), NGC 4102 (Jogee \& Kenney 1996), NGC 2782 (Jogee et al. 1997a, 1997b), and NGC 4536. In these galaxies, the circumnuclear molecular gas is concentrated over the steeply rising part of the rotation curve, and has predominantly circular motions in the region of peak star formation. In other galaxies (NGC 4569, NGC 6951, NGC 3351 (Kenney et al. 1992), and NGC 4314 (Benedict et al. 1996)), where large gas concentrations exist near or well beyond the turnover point of the rotation curve, $t_{\mathbf{s f}}$ is an order of magnitude higher. We expect that some galaxies like NGC 4569, NGC 4102, and NGC 3351 might be related through evolutionary paths as shown in Fig. 1. In NGC 4569, a large fraction of the gas is still inflowing towards the inner kpc, shows non-circular motions of several hundred $\mathrm{kms}^{-1}$, and is not forming stars efficiently. Within NGC $4102, \mathrm{t}_{\mathrm{sf}}$ is low in 


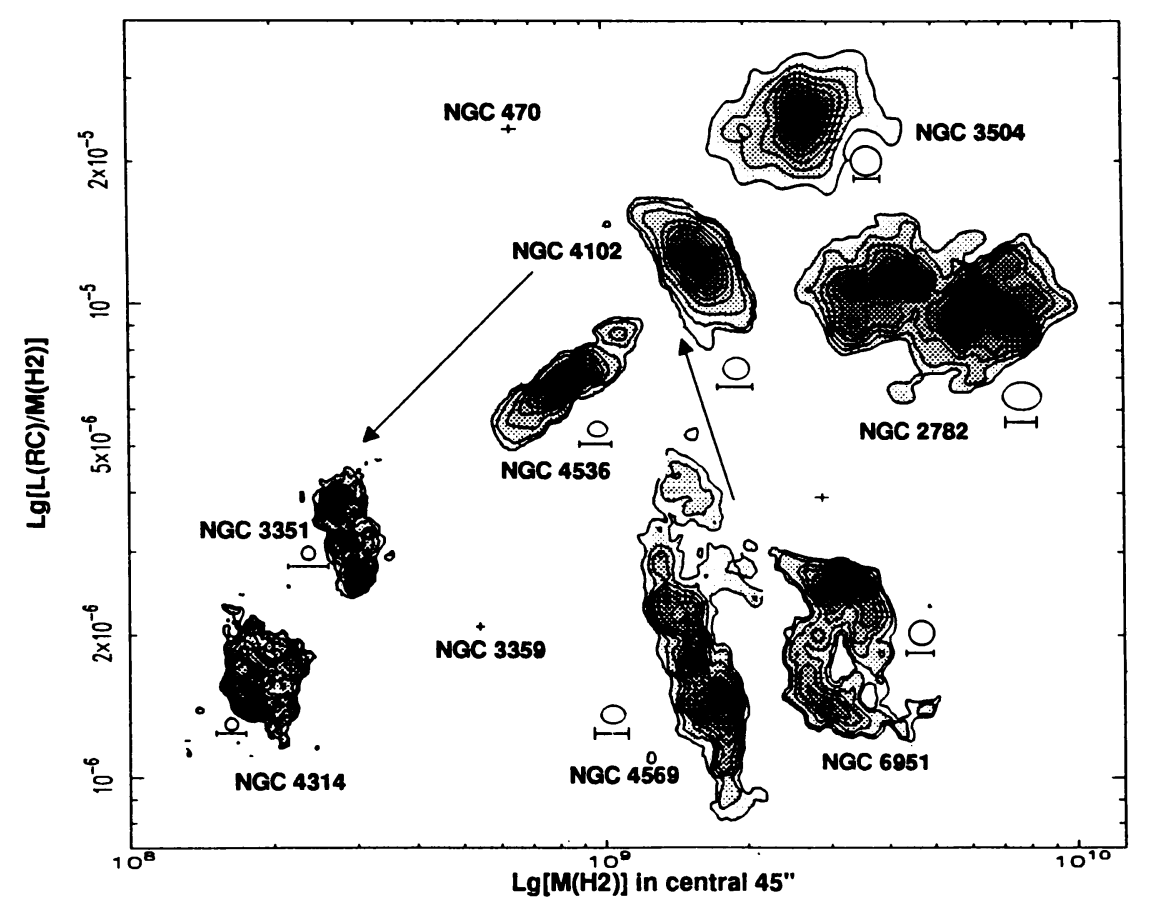

Figure 1. 2" CO maps from Jogee's thesis plotted in the $\mathrm{L}(\mathrm{RC}) / \mathrm{M}\left(H_{2}\right)$ versus $\mathrm{M}\left(H_{2}\right)$ plane (see text). Ellipses show the beamsizes, typically $2-3^{\prime \prime}$, and the horizontal line shows a scale of $300 \mathrm{pc}$. The arrows show likely evolutionary paths.

the inner $300 \mathrm{pc}$ where circular motions dominate (Jogee \& Kenney 1996), but further out it rises sharply by an order of magnitude. Thus, within less than a Gyr, the starburst is expected to transform the morphology from a wide $\mathrm{CO}$ annulus into a thin $\mathrm{CO}$ ring. This ring would be located near the turnover point of the rotation curve, have a large $t_{s f}$, and surround a young, massive, compact stellar disk. It is relevant that in NGC 3351, a disk-like component rather than a bulge appears to dominate the stellar light inside such a CO and $\mathrm{H} \alpha$ ring (Kenney \& Jogee 1998, in prep.).

\section{References}

Benedict, G. F., Smith, B. J., \& Kenney, J. D. P., 1996, AJ, 111, 1861

Condon, J. J., Helou, G., Sanders, D. B., \& Soifer, T. B. 1990, ApJS, 73, 359

Jogee, S., \& Kenney, J. D. P. 1996, in Proceedings of IAU Colloquium 157, eds. R. Buta,

D. A. Crocker, \& B. G. Elmegreen, (ASP Conference Series), p. 230

Jogee, S., Kenney, J. D. P., \& Smith, B. J. 1997a, ApJL, accepted.

Jogee, S., Kenney, J. D. P., \& Smith, B. J. 1997b, ApJ, submitted

Kenney, J. D. P., Carlstrom, J. E., \& Young, J. S. 1993, ApJ, 418, 687

Kenney, J. D. P., Wilson, C. D., Scoville, N. Z., et al. 1992, ApJ, 395, L79 